\title{
Tr-milRNA1 Contributes to Lignocellulase Secretion under Heat Stress by Regulating the Lectin-Type Cargo Receptor Gene Troip36 in Trichoderma guizhouence NJAU 4742
}

\author{
Tuo Li ${ }^{1}$, Jinding Liu ${ }^{2}$, Qin Wang ${ }^{1}$, Yang Liu ${ }^{1}$, Ting Li ${ }^{1}$, Dongyang Liu ${ }^{1} * * \mathbb{D}$ and Qirong Shen ${ }^{1}$ \\ 1 Jiangsu Provincial Key Lab of Solid Organic Waste Utilization, Jiangsu Collaborative Innovation Center of \\ Solid Organic Wastes, Educational Ministry Engineering Center of Resource-Saving Fertilizers, Nanjing \\ Agricultural University, Nanjing 210095, China; 2018203039@njau.edu.cn (T.L.); \\ 2020103111@stu.njau.edu.cn (Q.W.); 2021203046@stu.njau.edu.cn (Y.L.); 2020103119@stu.njau.edu.cn (T.L.); \\ shenqirong@njau.edu.cn (Q.S.) \\ 2 College of Information Management, Nanjing Agricultural University, Nanjing 210095, China; \\ liujd@njau.edu.cn \\ * Correspondence: liudongyang@njau.edu.cn
}

check for updates

Citation: Li, T.; Liu, J.; Wang, Q.; Liu, Y.; Li, T.; Liu, D.; Shen, Q.

Tr-milRNA1 Contributes to

Lignocellulase Secretion under Heat Stress by Regulating the Lectin-Type Cargo Receptor Gene Trvip36 in Trichoderma guizhouence NJAU 4742. J. Fungi 2021, 7, 997. https://doi.org/ $10.3390 /$ jof7120997

Academic Editor: Baojun Xu

Received: 7 October 2021

Accepted: 20 November 2021

Published: 23 November 2021

Publisher's Note: MDPI stays neutral with regard to jurisdictional claims in published maps and institutional affiliations.

Copyright: (c) 2021 by the authors. Licensee MDPI, Basel, Switzerland. This article is an open access article distributed under the terms and conditions of the Creative Commons Attribution (CC BY) license (https:// creativecommons.org/licenses/by/ $4.0 /)$.

\begin{abstract}
Background: MicroRNA plays an important role in multifarious biological processes by regulating their corresponding target genes. However, the biological function and regulatory mechanism of fungal microRNA-like RNAs (milRNAs) remain poorly understood. Methods: In this study, combined with deep sequencing and bioinformatics analysis, milRNAs and their targets from Trichoderma guizhouence NJAU 4742 were isolated and identified under solid-state fermentation (SSF) by using rice straw as the sole carbon source at $28{ }^{\circ} \mathrm{C}$ and $37{ }^{\circ} \mathrm{C}$, respectively. Results: A critical milRNA, TGA1_S04_31828 (Tr-milRNA1), was highly expressed under heat stress $\left(37^{\circ} \mathrm{C}\right)$ and adaptively regulated lignocellulase secretion. Overexpression of $T r$-milRNA1 (OE-Tr-milRNA1) did not affect vegetative growth, but significantly increased lignocellulose utilization under heat stress. Based on the bioinformatics analysis and qPCR validation, a target of Tr-milRNA1 was identified as Trvip36, a lectin-type cargo receptor. The expression of Tr-milRNA1 and Trvip36 showed a divergent trend under SSF when the temperature was increased from $28^{\circ} \mathrm{C}$ to $37^{\circ} \mathrm{C}$. In addition, the expression of Trvip36 was suppressed significantly in Tr-milRNA1 overexpression strain (OE-Tr-milRNA1). Compared with the wild type, deletion of Trvip36 (DTrvip36) significantly improved the secretion of lignocellulases by reducing the retention of lignocellulases in the ER under heat stress. Conclusions: Tr-milRNA1 from NJAU 4742 improved lignocellulose utilization under heat stress by regulating the expression of the corresponding target gene Trvip36. These findings might open avenues for exploring the mechanism of lignocellulase secretion in filamentous fungi.
\end{abstract}

Keywords: lignocellulase secretion; Trichoderma guizhouence NJAU 4742; heat stress; milRNA; posttranscriptional regulation

\section{Introduction}

Small RNA (sRNA)-induced RNA interference (RNAi) is a broad biological process that can lead to sequence-specific degradation or translational repression of target $\mathrm{mR}$ NAs [1,2]. The common feature of RNAi pathways is that sRNAs bind to Argonaute (AGO) proteins and guide the RNA-induced silencing complex (RISC) to RNAs with complementary sequences $[3,4]$. RNAi is highly conserved in eukaryotes, including the majority of the fungal kingdom [5]. Fungal RNA interference was first discovered in Neurospora crassa. Introducing fragments of albino-1 (al-1) or albino-3 (al-3), which are required for carotenoid biosynthesis, reduced al-1, or al-3 mRNA levels and resulted in an albino phenotype [6]. With the expansion of fungal genome information, RNAi pathway components have been found in most fungal species. Studies on Schizosaccharomyces pombe and other fungi have 
revealed various small RNA biogenesis pathways, suggesting that RNAi related pathways are utilized in all cellular processes to adapt to complex external environments [7].

sRNAs, defined by their length of 18-24 nucleotides, play significant roles in growth and development processes, pathogenicity and stress response $[2,8,9]$. Usually, miRNAs are a class of interior sRNAs found in eukaryotes, that function in many processes, such as development, biotic and abiotic reactions, and defense [10]. Previously, miRNA-like RNAs (milRNAs), with similar characteristics of miRNAs in animals and plants, were recognized and corroborated to be generated through at least four diverse pathways in $N$. crassa [11]. Interestingly, at least four distinct mechanisms have been discovered to produce milRNAs. Dicer-independent small interfering RNAs (disiRNAs) with a size of 21 or $22 \mathrm{nt}$ were also identified in Neurospora [11]. Afterward, a large number of milRNAs were identified in fungi. Fifteen milRNAs could regulate mycelium growth and conidiogenesis processes in Metarhizium anisopliae [12], and 27 sRNAs were found to have miRNA-like precursor structures in Botrytis cinerea [13]. In addition, the presence and differential expression of Trichoderma reesei milRNAs when T. reesei was cultivated in basal medium supplemented with $3 \%$ Avicel (microcrystalline cellulose powder) or $2 \%$ glucose, implied that milRNA might function in T. reesei growth and cellulase induction [14]. Nevertheless, analogous studies have mainly focused on bioinformatics predictions, but the biogenesis and biological function of milRNAs are rarely investigated.

Trichoderma guizhouense NJAU 4742, a saprophytic filamentous fungi isolated from mature compost, has recently attracted wide attention due to its potential to promote plant growth $[14,15]$. Similar to other heterotrophs, NJAU 4742 usually relies on particular host organisms or substrates for their nutrition. During the colonization process in different habitats including soils and plant roots, NJAU 4742 always feeds on dead fungi or efficiently degrades different plant debris to obtain enough energy resources [16]. Thus, the synthesis and secretion of lignocellulases by NJAU 4742 is one of the critical parameters during the colonization process. Nevertheless, various factors affect its biological functions, especially when the topsoil temperature is too high. Our previous results showed that the secretion of lignocellulases by NJAU 4742 was significantly suppressed at $37^{\circ} \mathrm{C}$ (unpublished results). Here, deep sequencing and molecular assays were used to reveal the mechanism of lignocellulase secretion in NJAU 4742 under posttranscriptional regulation mediated by Tr-milRNAs. The preliminary analysis of the results indicated that TGA1_S04_31828 (Tr-milRNA1) adaptively regulates a lectin-type cargo receptor (Trvip36), which might be related to the lignocellulase secretion under heat stress. VIP36 is an intracellular lectin cycling between the endoplasmic reticulum (ER) and the Golgi apparatus, and it is deemed to serve as a cargo receptor in the transport and classification of glycoproteins [17]. VIP36 has also been shown to recycle human $\alpha 1$-antitrypsin from the Golgi compartment back to the ER, and silencing VIP36 improved $\alpha 1$-antitrypsin production. This fact demonstrates that this cargo receptor has a protein retention function [18]. In Aspergillus oryzae, deletion of VIP36 improved heterologous protein secretion, and the results of ER-enriched cellular fractions revealed that VIP36 was involved in the retention of heterologous proteins in the ER [19]. As VIP36 could form a stable complex with the molecular chaperone BiP, it appeared to be involved in the quality control of secretory proteins [17]. Therefore, lectin-type cargo receptors might alter the secretion of cellulases, which would be potential suitable models for studying the relationship between cargo receptors and secretory proteins in filamentous fungi.

The objective of this study was to uncover the role of Tr-milRNA in lignocellulose utilization of NJAU4742 under heat stress. Further studies investigated the Tr-milRNA1 regulation mechanism and the involvement of lectin-type cargo receptors in the intracellular transfer of cellulases in NJAU 4742 under heat stress, which aids readers to have a deeper understanding of lignocellulose biodegradation by filamentous fungi. 


\section{Materials and Methods}

\subsection{Strains and Culture Conditions}

T. guizhouense NJAU 4742 isolated from a compost sample was maintained in our laboratory, and its genome sequence was deposited in the NCBI database (Accession No. LVVK00000000.1). Mandels' salt solution without organic components (1.4 $\mathrm{g} \mathrm{L}^{-1}$ $\left(\mathrm{NH}_{4}\right)_{2} \mathrm{SO}_{4}, 2.0 \mathrm{~g} \mathrm{~L}^{-1} \mathrm{KH}_{2} \mathrm{PO}_{4}, 0.3 \mathrm{~g} \mathrm{~L}^{-1} \mathrm{CaCl}_{2}, 0.3 \mathrm{~g} \mathrm{~L}^{-1} \mathrm{MgSO}_{4}, 5 \mathrm{mg} \mathrm{L}-1 \mathrm{FeSO}_{4} \cdot 7 \mathrm{H}_{2} \mathrm{O}$, $20 \mathrm{mg} \mathrm{L}^{-1} \mathrm{CoCl}_{2}, 1.6 \mathrm{mg} \mathrm{L}^{-1} \mathrm{MnSO}_{4}$ and $\left.1.4 \mathrm{mg} \mathrm{L}^{-1} \mathrm{ZnSO}_{4}\right)$ supplemented with $2 \%(w / v)$ rice straw was used for lignocellulase production under SSF [20]. Spores were obtained from incubated PDA plates followed by filtration with four layers of sterilized gauze, and the spore suspension was adjusted to $1.0 \times 10^{7}$ spores $\cdot \mathrm{mL}^{-1}$ by quantifying spores on a hemocytometer.

\subsection{Small RNA cDNA Library Construction and High-Throughput Sequencing}

Mycelial samples were harvested after $72 \mathrm{~h}$ by using rice straw as the sole carbon source at $28^{\circ} \mathrm{C}$ (T28) and $37^{\circ} \mathrm{C}$ (T37). Total RNA was extracted using the RNeasy ${ }^{\circledR}$ Plant Mini Kit (Qiagen, Hilden, Germany) and handled with DNase I (TaKaRa) by following the manufacturer's instructions. RNA concentration and purity were evaluated in an Agilent 2100 Bioanalyzer (Agilent, Santa Clara, CA, USA) to check RNA integrity. Sequencing libraries were generated by employing NEBNext ${ }^{\circledR}$ Multiplex Small RNA Library Prep Set for Illumina ${ }^{\circledR}$ (New England BioLabs Inc., Beverly, MA, USA) according to the manufacturer's instructions. The clustering of each index-coded sample was carried out on a cBot Cluster Generation System using TruSeq SR Cluster Kit v3-cBot-HS (Illumina, San Diego, CA, USA) according to the manufacturer's instructions. Finally, libraries were sequenced through an Illumina HiSeq 2500 platform.

\subsection{Tr-milRNAs Sequence Analysis and Target Prediction}

The original reads from sequencing data were filtered by removing poor quality reads, adaptor contamination reads and reads longer than $30 \mathrm{nt}$ or shorter than $18 \mathrm{nt}$. The standard-compliant reads of small RNAs were aligned to the reference NJAU 4742 genome (https://bioinfo.njau.edu.cn/tgn4742/, accessed on 20 November 2021). The alignment analysis was performed on the CLC Genomics Workbench 12. The sequences that corresponded to known miRNAs were defined by matching to the miRNA database (miRBase 22.0) [21]. The unannotated sRNA sequences were aligned to the reference NJAU 4742 genome to identify precursor sequences for novel miRNAs. Novel miRNAs were predicted by miRDeep2 with a stem-loop structure [22]. The R package DEGseq software was used to analyze the differentially expressed miRNAs. TargetFinder and psRNATarget were used to predict the candidate target genes of Tr-milRNAs [23,24].

\subsection{Relative Expression of Tr-milRNA1 and the Corresponding Target Genes}

Samples of different treatments were collected at 0, 24, 36, 48, 60 and $72 \mathrm{~h}$ post inoculation (hpi). Total RNA was extracted using the RNeasy ${ }^{\circledR}$ Plant Mini Kit (Qiagen, Germany) according to the manufacturer's instructions. Expression of Tr-milRNA1 was determined by stem-loop qRT-PCR as previously described [25]. First strand cDNA was synthesized by miRNA First Strand cDNA Synthesis (Vazyme Biotech, Nanjing, China) with the stem-loop RT primer based on the manufacturer's instructions. PCR detection was performed by a Tr-milRNA1-specific forward primer and a universal reverse primer. The NJAU 4742 18S rRNA biogenesis gene (18S) was used as a control. qRT-PCR was performed using the miRNA Universal SYBR qPCR Master Mix (Vazyme Biotech, Nanjing, China) according to the manufacturer's instructions. For the determination of transcript levels of the corresponding target genes, cDNA synthesis was completed by the PrimeScript RT Reagent Kit (RR036A, Takara, Dalian, China) according to the manufacturer's instructions. qRT-PCR was performed using SYBR Premix Ex Taq II (RR820A, Takara, Dalian, China) and the CFX connect ${ }^{\mathrm{TM}}$ Real-Time system (Bio-Rad, Hercules, CA, USA). Transcription levels of the target genes were normalized by the $2^{-\Delta \mathrm{Ct}}$ method, and translation elongation 
Factor 1 alpha (Tef) was used as the housekeeping gene. All primers used in this study are listed in Table S1.

\subsection{Deletion and Overexpression of Fungal Small RNAs}

For targeted deletion of $T r$-milRNA1, the $5^{\prime}$ and $3^{\prime}$ flanking regions of $T r$-milRNA1 were amplified by PCR from NJAU 4742 genomic DNA. HygB was used as a resistance gene and the fragment was amplified by PCR from plasmid pcDNA1 (Vienna University of Technology). The three fragments were fused by CloneAmp HiFi PCR Premix (Takara, Japan) according to the manufacturer's instructions. The Tr-milRNA1 deletion mutant was generated via a gene replacement strategy using the polyethylene glycol (PEG)-mediated protoplast transformation procedure as described in Zhang et al. [26].

To overexpress $T r$-milRNA1 in NJAU 4742, the $400 \mathrm{bp}$ fragment surrounding primary $T r$-milRNA1 (a fragment of approximately $200 \mathrm{bp}$ upstream and downstream of primary TrmilRNA1) was amplified by PCR from NJAU 4742 genomic DNA and then introduced into plasmid pcDNA1 by the ClonExpress-II One Step Cloning Kit (Vazyme Biotech, Nanjing, China). In pcDNA1: Tr-milRNA1 precursor construct was expressed under the control of the cDNA1 promoter (Figure S1b). Mutated Tr-milRNA1 (OE-Mut-Tr-milRNA1) expression construct was obtained by a Fast Site-Directed Mutagenesis Kit (Tiangen) according to the manufacturer's instructions. The sequence of constructs was confirmed by sequencing (Tongyong Biological Technology, Chuzhou, China). The vectors of OE-Tr-milRNA1, OEMut-Tr-milRNA1 and empty vector were separately transformed into NJAU 4742 by using the polyethylene glycol (PEG)-mediated protoplast transformation procedure [26]. The relative expression level of Tr-milRNA1 between wt, mutants OE-Tr-milRNA1, OE-Mut-TrmilRNA1 and empty vector transformant (EV) was measured following the above method (relative expression of $T r$-milRNA1 at different sampling times). A diagram shows the strategy of Tr-milRNA editing and mutant detection used in this study (Figure S1a,b,d,f,g), and the primers are given in Table S1.

\subsection{Generation of the Target Gene Mutants}

To obtain single knockout mutant homologous recombination fragments of Trvip36, the $5^{\prime}$ and $3^{\prime}$ flanking regions of the gene open-reading frame (ORF) were amplified by PCR from NJAU 4742 genomic DNA, and the two fragments were ligated with Hygb through CloneAmp HiFi PCR Premix (Clontech) according to the manufacturer's instructions. To generate a Trvip36 overexpression mutant homologous recombination fragment, three fragments including the $5^{\prime}$ flanking regions of the gene ORF, the promoter fragment and the ORF of the Trvip36 gene fragment were amplified by PCR from NJAU 4742 genomic DNA and then fused with Hygb by using CloneAmp HiFi PCR Premix (Clontech). Trvip36 deletion and overexpression mutants were generated by using homologous recombination and a polyethylene glycol (PEG)-mediated protoplast transformation system [26]. A diagram shows the strategy of gene editing and mutant detection used in this study (Figure S1a,c,e), and the primers are given in Table S1.

\subsection{Growth and Enzyme Activity Assays of NJAU 4742}

Equally harvested biomass samples of different treatments were transferred to the medium with rice straw as the sole carbon source and incubated at $37{ }^{\circ} \mathrm{C}$ to determine the growth rate. For enzyme activity assays, $1 \mathrm{~mL}$ of fresh spore suspension $\left(1.0 \times 10^{7}\right.$ spores $\left.\cdot \mathrm{mL}^{-1}\right)$ of different strains was inoculated for SSF. All samples of different treatments were collected on the 4th day, and three biological replicates were collected at each sampling point. Filter paper activity (FPA) and endoglucanase activity (EG) were measured according to the method described by Xue et al. [27] with filter paper (Whatman NO.1) and CMC-Na (Sigma, St. Louis, MO, USA) as the substrates. Xylanase activity (XYL) was assayed with oat spelts xylan (Sigma, St. Louis, MO, USA) as the substrate [28]. The reaction system was executed in $0.1 \mathrm{M}$ acetate buffer $(\mathrm{pH} 4.8)$ at $50{ }^{\circ} \mathrm{C}$ for $10 \mathrm{~min}$, after which the DNS method was used to measure the released reducing sugars. The 
cellobiohydrolase activity $(\mathrm{CBH})$ was determined in $0.1 \mathrm{M}$ acetate buffer at $50{ }^{\circ} \mathrm{C}$ for $30 \mathrm{~min}$ with pNPC (Sigma, St. Louis, MO, USA) as the substrate according to Liu et al. [29]. One enzyme activity unit was defined as the amount of enzyme required to liberate $1 \mu \mathrm{mol}$ glucose or pNP per minute under the assayed conditions.

\subsection{Confocal Imaging of EGL-GFP and CBH-GFP in the ER and Golgi Apparatus between wt and $\Delta$ Trvip36}

The DNA fractions of the representative lignocellulase genes including endoglucanase (egl, A1A110863.1) and cellobiohydrolase (cbh, A1A102028.1) were amplified by DNA of NJAU 4742, and the eGFP fragment was amplified by using plasmid pEGFP-N1 (Clontech, Mountain View, CA, USA). The DNA fractions of different cellulase genes were ligated with the GFP fragment by the overlapping-PCR technique based on the instructions of CloneAmp HiFi PCR Premix (Clontech, Mountain View, CA, USA). Mutants of endoglucanase-GFP (egl-GFP) and cellobiohydrolase-GFP ( $c b h-G F P)$ were obtained through homologous recombination based on wt and $\Delta$ Trvip36 (Figures S1h and S2). Subsequently, these mutants labeled with GFP in situ were cultured in medium with rice straw as the sole carbon source for three days, and the freshly cultured mycelium was incubated with ER-Tracker ${ }^{\mathrm{TM}}$ Red (Thermo Fisher Scientific, Cat. M7512, Ex/Em = $587 \mathrm{~nm} / 615 \mathrm{~nm}$ ) or the BODIPY $^{\mathrm{TM}}$ TR Ceramide (Thermo Fisher Scientific, D7540, Ex $/ \mathrm{Em}=589 \mathrm{~nm} / 616 \mathrm{~nm}$ ) at a concentration of $100 \mathrm{nM}$ at room temperature for $5 \mathrm{~min}$ in darkness. Subsequently, the cellulase-GFP and ER or Golgi apparatus stained hyphae were imaged under a confocal fluorescence microscope (TCS SP8, Leica, Germany), and the fluorescence intensity and colocalization analysis were performed by plot profile in ImageJ software according to Zhao et al. [30]. All fluorescence intensity values of EGL-GFP and CBH-GFP in the ER and Golgi apparatus were counted when the fluorescence intensity value of ER-Tracker or Golgi-Tracker was valid (higher than 0). Data were completed as at least three independent biological replicates. Statistical data were expressed as means \pm standard errors (SE) from all valid pixels.

\section{Results}

\subsection{Identification and Quantification of milRNAs in NJAU 4742}

In order to determine whether milRNAs are involved in the regulation of cellulase secretion in NJAU 4742 under heat stress, two small RNA libraries were generated from NJAU 4742, grown using rice straw rice straw as the sole carbon source at $28{ }^{\circ} \mathrm{C}(\mathrm{T} 28)$ and $37^{\circ} \mathrm{C}$ (T37), respectively. In total, 56 putative milRNAs were identified in NJAU 4742, and 47 and 46 Tr-milRNAs were identified at T28 and T37, respectively (Table 1 and Figure 1a). $\mathrm{Tr}$-milRNAs were enriched at $22 \mathrm{nt}$ and seemed to possess a strong preference for uracil (Figure 1b,c). Interestingly, 10 and 9 Tr-milRNAs were specifically expressed at T28 or T37, respectively. Thirty-seven Tr-milRNAs were coexpressed in T28 and T37.

After normalization, $20 \mathrm{Tr}$-milRNAs with transcripts per million (TPM) values higher than 10 were identified. TGA1_S20_236772 and TGA1_S20_238808 exhibited a high abundance at $28^{\circ} \mathrm{C}$. In contrast, TGA1_S04_31828 (Tr-milRNA1), TGA1_S02_9056, TGA1_S05_43489 (Tr-milRNA2), TGA1_S06_56271 (Tr-milRNA3), TGA1_S10_93851 (Tr-milRNA4), TGA1_S17_ 158248 (Tr-milRNA5), TGA1_S17_162471, TGA1_S17_175238, TGA1_S17_175348, TGA1_S18_ 185930 (Tr-milRNA6), TGA1_S19_204554, TGA1_S20_223477, TGA1_S20_238860, TGA1_S2 0_238862,TGA1_S22_248446, TGA1_S22_248536, TGA1_S22_253299,TGA1_S22_257134 and TGA1_S30_293034 showed a high abundance at $37^{\circ} \mathrm{C}$ (Table 1 and Figure $\left.1 \mathrm{~d}\right)$. At a false discovery rate $<0.05$ and $\log _{10}$ fold change $>1$ or $<-1$, six significantly upregulated $\mathrm{Tr}$ milRNAs including Tr-milRNA1, Tr-milRNA2, Tr-milRNA3, Tr-milRNA4, Tr-milRNA5 and Tr-milRNA6 were selected (Figure 1e). This result indicated that at least several Tr-milRNAs might be involved in the regulation of lignocellulose utilization at different temperatures. 
Table 1. Identification and expression abundance of milRNAs in NJAU 4742 by miRNA deep sequencing.

\begin{tabular}{|c|c|c|c|c|c|}
\hline miRNA_ID & Mirdeep2_Score & RNAfold_Results & TPM (T28) & TPM (T37) & Mature_Sequence \\
\hline TGA1_S20_236772 & $2,295,965.4$ & yes & $983,492.79$ & $976,779.71$ & uuuuugagauacuccgcaacgac \\
\hline TGA1_S20_238860 & $31,534.5$ & yes & $11,201.07$ & $16,331.53$ & uacguaggacuuuaccgugacgu \\
\hline TGA1_S06_56271 & 115.5 & yes & 12.30 & 112.44 & uugccgaguggcagaggacuggcau \\
\hline TGA1_S17_175348 & 105 & yes & 23.71 & 47.53 & uggaaguugaaucgagaagcccu \\
\hline TGA1_S17_158248 & 85.8 & yes & 6.96 & 89.12 & gcaucugauuuccacccuuggguu \\
\hline TGA1_S19_204554 & 43.5 & yes & 10.77 & 28.55 & agcuuuuggcuuuccagaacccgu \\
\hline TGA1_S19_204542 & 38 & yes & 11.73 & 11.75 & uggcuggacggccagagggccu \\
\hline TGA1_S30_293034 & 14.6 & yes & 2.86 & 35.64 & cgcaggcucgauuguugucu \\
\hline TGA1_S14_132594 & 13.2 & yes & 0.18 & 4.88 & cgccaacggccucgeceggcuac \\
\hline TGA1_S19_188258 & 12.5 & yes & 2.18 & 2.91 & caucugcauguugucucugg \\
\hline TGA1_S10_93851 & 11.9 & yes & 0.56 & 18.67 & gucuagcacucuacuuuggcau \\
\hline TGA1_S17_156622 & 9.3 & yes & 0.30 & 6.57 & uuuuuacacagauaccaguaggu \\
\hline TGA1_S20_237911 & 7.5 & yes & 0.77 & 5.62 & ccaccaggccgcucaagacua \\
\hline TGA1_S03_23817 & 7.1 & yes & 0.68 & 5.62 & cacugaccugcuccucgcacag \\
\hline TGA1_S17_161581 & 7.1 & yes & 0 & 1.69 & caucuagcuuggacggcagcg \\
\hline TGA1_S11_99749 & 6.5 & yes & 0.30 & 5.62 & ucgucaccuugaggcggaa \\
\hline TGA1__S02_7777 & 5.4 & yes & 0.86 & 3.66 & auugcgaugccuggucagcuacuc \\
\hline TGA1_S22_248536 & 5.4 & yes & 0.38 & 12.40 & ugaggagucugaagauggagaggaa \\
\hline TGA1_S03_27309 & 5.3 & yes & 0.60 & 7.80 & guggacagauuagcugacccgcggg \\
\hline TGA1_S15_136221 & 4.5 & yes & 0.36 & 0 & accaacagcggacauugcuccaa \\
\hline TGA1_S11_101486 & 4 & yes & 0.60 & 0 & ugcuccaaaugagaaucgaagu \\
\hline TGA1_S11_106603 & 3.7 & yes & 0.76 & 0 & ugccugguacauguacgga \\
\hline TGA1_S07_71495 & 3.5 & yes & 1.80 & 0.85 & ugaccaagaacuucgacgucuu \\
\hline TGA1_S15_142991 & 3.3 & yes & 0 & 2.54 & uuuggaccgugucuggaacgcua \\
\hline TGA1_S17_178322 & 3.3 & yes & 0 & 5.62 & ucauauucucagcacuuggaau \\
\hline TGA1_S02_16357 & 3.2 & yes & 0 & 1.70 & agguaacgucugguggcaa \\
\hline TGA1_S05_35063 & 2.9 & yes & 0 & 4.98 & gcaagaucaaaacucaaa \\
\hline TGA1_S17_175238 & 2.8 & yes & 4.34 & 13.62 & uggaaguugaaucgagaagccc \\
\hline TGA1_S09_88615 & 2.7 & yes & 0 & 1.70 & cguguaccagagcgucau \\
\hline TGA1_S08_81282 & 2.5 & yes & 0.60 & 0 & uccgauagggauguucgggcu \\
\hline TGA1_S20_238862 & 2.5 & yes & 4634.89 & 4921.04 & uaggacuuuaccgugacgucc \\
\hline TGA1_S22_257134 & 2.5 & yes & 1.45 & 12.20 & cgguggauugaacgggacucuuggu \\
\hline TGA1_S05_43489 & 2.4 & yes & 28.74 & 491.72 & cucgggagaaggcggccu \\
\hline TGA1_S07_78907 & 2.3 & yes & 0.36 & 0 & gucuucuugaucucuuau \\
\hline TGA1_S18_185930 & 2.3 & yes & 25.59 & 160.54 & accoggcuuggagaugug \\
\hline TGA1_S20_209761 & 2.3 & yes & 0.38 & 0 & ugaugauucggcuaguucggacag \\
\hline TGA1_S22_248446 & 2.3 & yes & 0.94 & 10.71 & cggcgaggcuguguuucagcga \\
\hline TGA1_S20_223477 & 2.2 & yes & 445.56 & 655.43 & uacguaggacucuaccgugacau \\
\hline TGA1_S20_238808 & 2.2 & yes & 44.36 & 38.21 & guucgaggguugaaauga \\
\hline TGA1_S06_55136 & 2 & yes & 0 & 1.70 & auguaugccuccugagauua \\
\hline TGA1_S16_151294 & 2 & yes & 0 & 1.70 & acuucauccauagauaucgcaa \\
\hline TGA1_S17_162471 & 2 & yes & 3.95 & 13.62 & uggaaguugaaucgagaagccc \\
\hline TGA1_S19_199524 & 1.9 & yes & 0.18 & 0 & uaccucucauucacugcagug \\
\hline TGA1_S02_9056 & 1.8 & yes & 1.77 & 26.56 & ggaaacaagguuguucugacuau \\
\hline TGA1_S13_112804 & 1.8 & yes & 0.60 & 0 & uuaugugguacggcagagagu \\
\hline TGA1_S04_31828 & 1.7 & yes & 0.30 & 23.35 & gguucgacucccggcuugu \\
\hline TGA1_S14_132761 & 1.6 & yes & 7.96 & 6.95 & aacgugcaauugcuaccaa \\
\hline TGA1_S20_229688 & 1.6 & yes & 2.62 & 5.62 & ugagggaccggauucgcca \\
\hline TGA1_S22_253299 & 1.6 & yes & 15.54 & 38.89 & auccaaaagcucggcuuu \\
\hline TGA1_S23_271212 & 1.5 & yes & 0 & 5.62 & agcgaggacauuuaugac \\
\hline TGA1_S05_33550 & 1.4 & yes & 2.47 & 27.03 & accuagagaacgaugguucccauu \\
\hline TGA1_S07_67387 & 1.3 & yes & 0.71 & 0 & aagcagauugcgaggggucauuug \\
\hline TGA1_S22_265703 & 1.1 & yes & 0.38 & 9.86 & gggcagucuguuggacuccggu \\
\hline TGA1_S07_76476 & 1 & yes & 1.14 & 0.85 & cucggucuguuguggauugguc \\
\hline TGA1_S19_189543 & 0.7 & yes & 1.14 & 0 & aaggacauuuuggaggag \\
\hline TGA1_S07_65040 & 0.1 & yes & 1.50 & 5.73 & ucgaaguugugguuguagugguagu \\
\hline
\end{tabular}


a
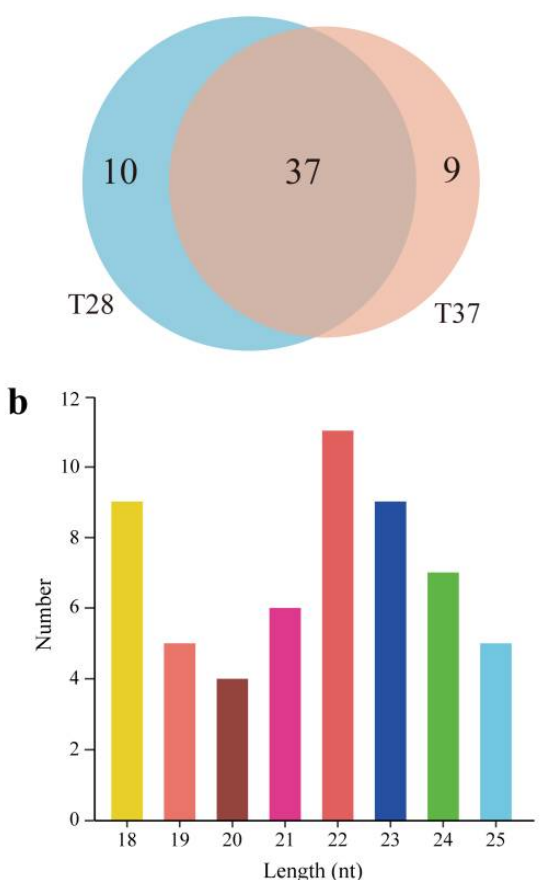
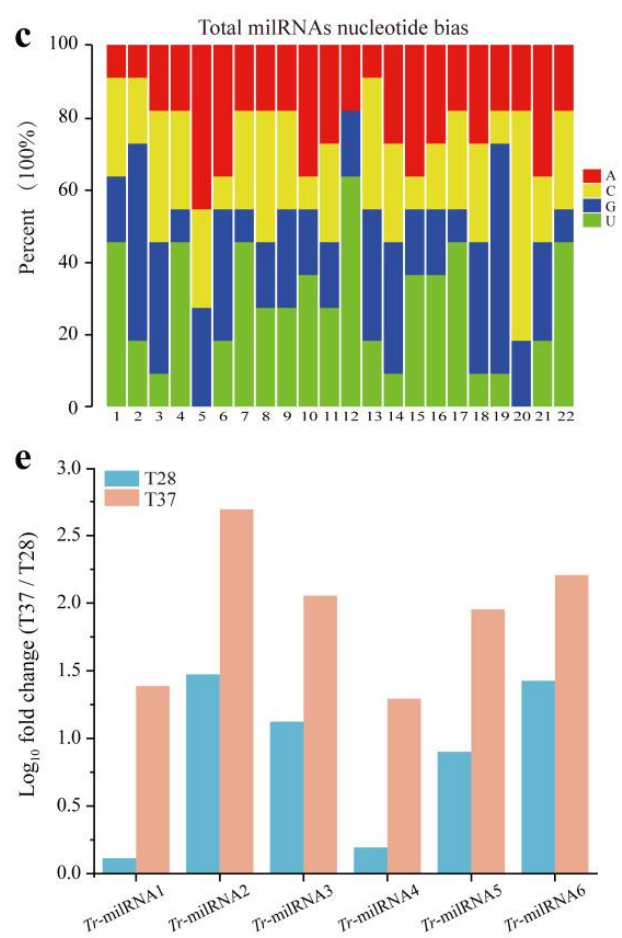

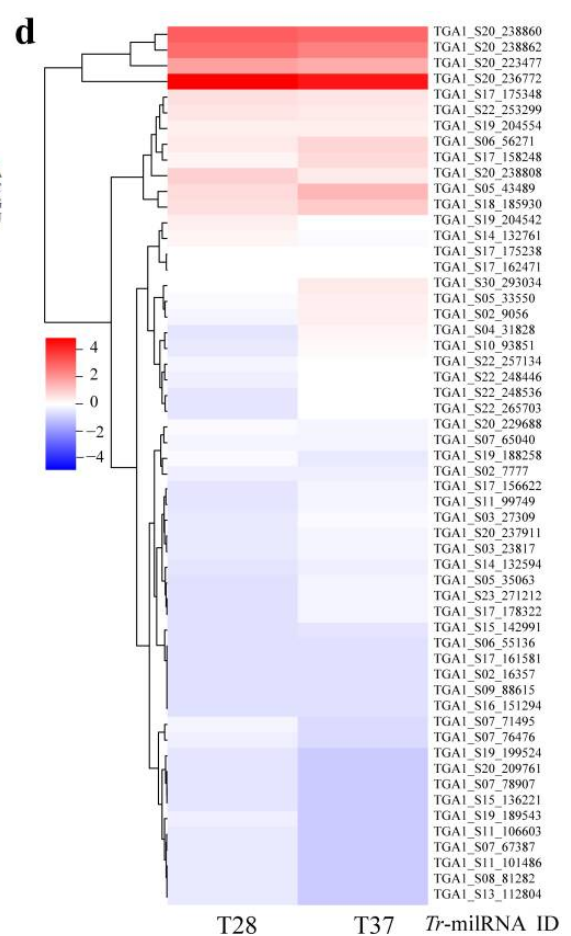

Figure 1. Tr-milRNAs are differentially expressed under SSF using rice straw as the sole carbon source at $28^{\circ} \mathrm{C}(\mathrm{T} 28)$ and $37^{\circ} \mathrm{C}$ (T37). (a) Different quantities of Tr-milRNAs were isolated from T28 (blue) and T37 (orange). Ten Tr-milRNAs and nine Tr-milRNAs are expressed specifically at T28 or T37, respectively. Thirty-seven Tr-milRNAs are coexpressed in T28 and T37; (b) length distribution of Tr-milRNAs; (c) nucleotide bias of Tr-milRNAs; (d) $\log _{10}$ (TPM) normalized values of all Tr-milRNAs were used to generate the heatmap. Red and blue colors indicate relatively high and low Tr-milRNA expression, respectively, and white equals median abundance; (e) normalized read numbers of Tr-milRNAs with transcripts per million (TPM) values higher than 10 in T28 or T37.

\subsection{Identification of Candidate Target Genes Related to Tr-milRNAs of NJAU 4742}

To investigate target genes of putative Tr-milRNAs, two rigorous target prediction software programs (TargetFinder and psRNATarget) were used to predict potential targets in the coding sequences, $3^{\prime}$ UTR and $5^{\prime}$ UTR of the NJAU 4742 genome. Gene ontology (GO) enrichment analysis indicated that candidate target genes were predictably involved in metabolic process, cellular process, single-organism process, catalytic activity, cell and membrane binding (Figure S3a). Tr-milRNA target genes also seem to be enriched through KEGG pathways including carbohydrate metabolism, catabolism, transport and translation (Figure S3b). To further ascertain whether the Tr-milRNA target genes were involved in the utilization of lignocellulose at different temperatures, putative target genes predictably involved in the secretion, synthesis and transportation of lignocellulases were selected. These findings might reveal that the differences in lignocellulose utilization at different temperatures are precisely regulated by Tr-milRNAs and their targets.

\subsection{Tr-milRNA1 Exhibits a Critical Role in Lignocellulose Utilization at Different Temperatures}

To explore the possible roles of $T r$-milRNAs in lignocellulose utilization at different temperatures, overexpression transformants of $T r$-milRNAs that were significantly upregulated at $37^{\circ} \mathrm{C}$ were generated to screen their function under heat stress. The results showed that $T r$-milRNA1 overexpression mutant (OE-Tr-milRNA1) could significantly increase lignocellulose utilization and also exhibited faster growth rate of mycelium using rice straw as the sole carbon source under heat stress than wt. Additionally, the pri-Tr-milRNA1 (primary transcript of $T r$-milRNA1) deletion mutant ( $\Delta T r$-milRNA1) exhibited normal growth, but showed a slight reduction in lignocellulose utilization capacity (Figure 2a). Various lignocellulosic enzyme activities were systematically assessed to determine the 
utilization efficiency of lignocellulose for filamentous fungi [31]. Therefore, different lignocellulases, including endoglucanase (EG), cellbiohydrolase (CBH), xylanase (XYL), and filter paper activity (FPA) were detected under SSF in different treatments. Noteworthy, EG and CBH activities increased significantly compared with wt under heat stress $(p<0.05)$. Correspondingly, various lignocellulosic enzyme activities of $\Delta T r$-milRNA1 decreased at different levels compared to wt (Figure 2b). These findings suggested that $T r$-milRNA1 might be critical in regulating the utilization of lignocellulose at different temperatures.

a

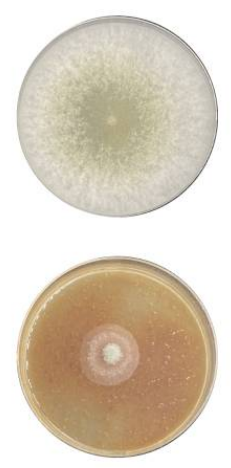

$\Delta \operatorname{Tr}$-milRNA1
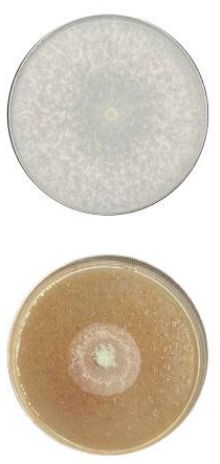

wt
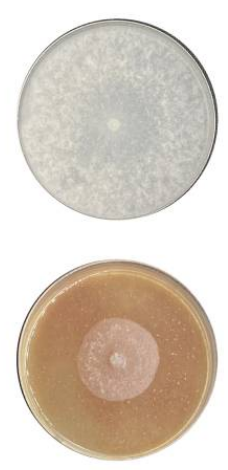

OE-Tr-milRNA1
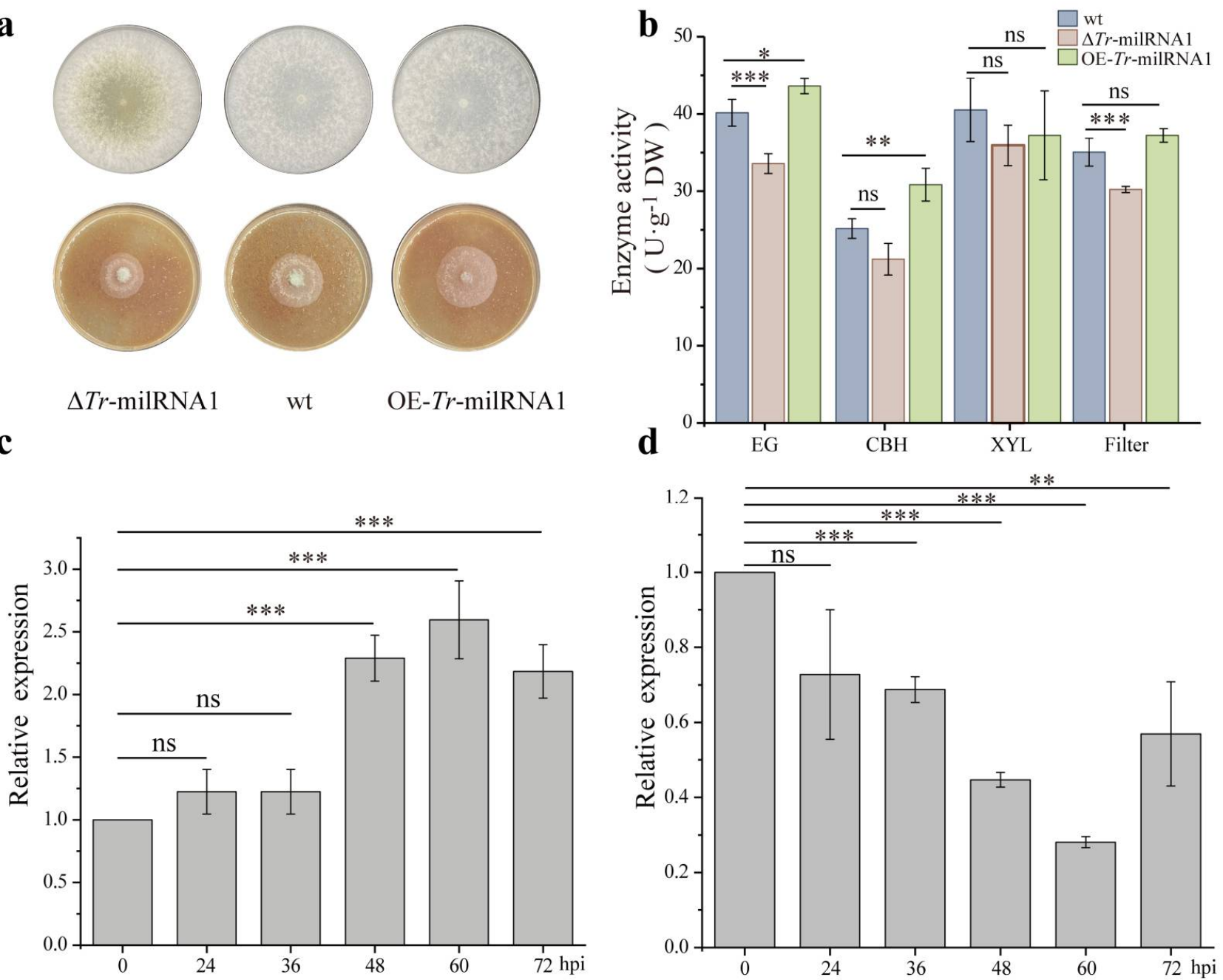

Figure 2. Overexpression of Tr-milRNA1 increased cellulase activities by silencing the target gene Trvip36. (a) Biomass comparison of wt and mutants on PDA at $28^{\circ} \mathrm{C}$ (top row) and rice straw medium at $37^{\circ} \mathrm{C}$ (bottom row); (b) the hydrolase activities, including FPA, EG, CBH and XYL of wt and mutants by using rice straw as the sole carbon source under heat stress; (c) relative expression levels of $\mathrm{Tr}$-milRNA1 from 0 to $72 \mathrm{hpi}$ at $37^{\circ} \mathrm{C}$. Relative expression levels of $\mathrm{Tr}$-milRNA1 were normalized to gene $18 \mathrm{~S}$ rRNA and calibrated to the levels of wt at 0 hpi (set as 1.0). (d) relative expression levels of target Trvip36 from 0 to $72 \mathrm{hpi}$ at $37^{\circ} \mathrm{C}$. Relative expression levels of Trvip36 were normalized to the Tef gene and calibrated to the levels of wt at $0 \mathrm{hpi}$ (set as 1). Data were calculated from three biological replicates, and error bars represent \pm SDs. ${ }^{*} p<0.05,{ }^{* *} p<0.01,{ }^{* * *} p<0.001$. A $p$ value $<0.05$ was regarded as statistically significant and ns refers to no significance.

\subsection{Expression of Troip36 Could Be Regulated by Tr-milRNA1}

Among the preselected target genes of Tr-milRNA1, we found that $T r$-milRNA1 could base pair with several genes predictably related to lignocellulose utilization, including genes encoding carbohydrate hydrolases, transport proteins, and energy homeostasis related proteins. To determine the interaction between $T r$-milRNA1 and the candidate target genes, we performed target verification tests. The relative expression of Tr-milRNA1 
and predicted target genes were determined using stem-loop qRT-PCR and qRT-PCR to determine if the expression levels of $T r$-milRNA1 and candidate target genes are correlated. During SSF, Tr-milRNA1 was upregulated at 36, 48, and $60 \mathrm{hpi}$ and downregulated at $72 \mathrm{hpi}$ (Figure 2c). Interestingly, we found that the transcript levels of the lectin-type cargo receptor gene Trvip 36 were downregulated at 0 to $60 \mathrm{hpi}$ and then upregulated at $72 \mathrm{hpi}$ (Figure 2d). However, the results for the other predicted target genes were illogical and contradictory. Thus, the negatively correlated expression of Tr-milRNA1 and Trvip36 might indicate that Trvip36 could be target of Tr-milRNA1.

\subsection{Tr-milRNA1 Regulates Target Gene Expression in a Sequence-Specific Manner}

Tr-milRNA1 had a $64 \mathrm{bp}$ putative precursor, which might form a typical hairpin structure (Figure 3a). To confirm the regulatory mechanism of Tr-milRNA1, a mutated $T r$-milRNA1 overexpression transformant (OE-Mut-Tr-milRNA1) was generated by sitespecific mutagenesis (Figure 3b). Subsequently, the transcript level of Trvip36 was quantified in wt, OE-Tr-milRNA1, OE-Mut-Tr-milRNA1, and EV at $60 \mathrm{hpi}$ under SSF. Interestingly, the expression of Trvip36 was significantly suppressed in OE-Tr-milRNA1, but not in OEMut-Tr-milRNA1 or EV (Figure 3c). These results suggested that Tr-milRNA1 might regulate the expression of Trvip36 in a sequence-specific manner.

$\mathbf{a}$

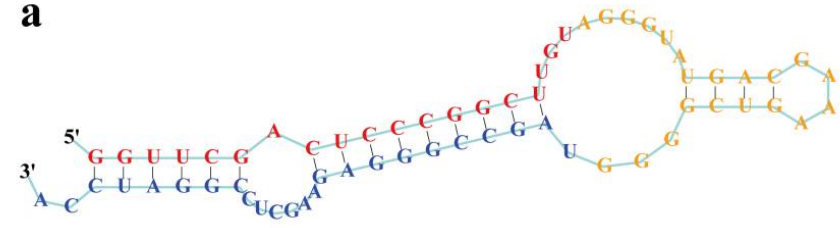

b

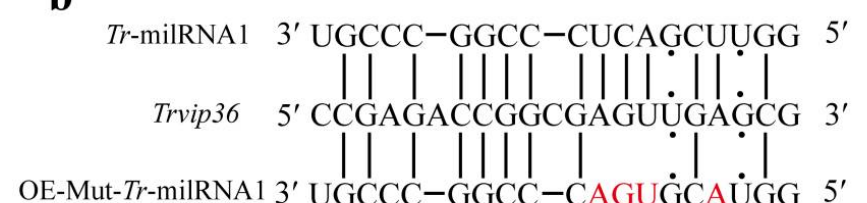

d

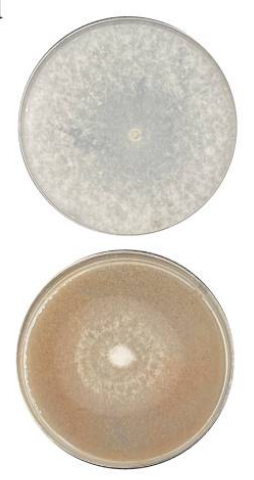

$\Delta$ Trvip36

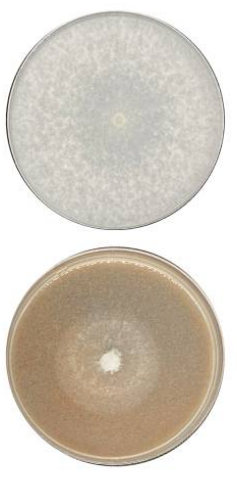

wt

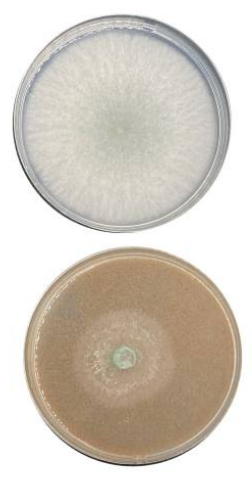

OE-Trvip36

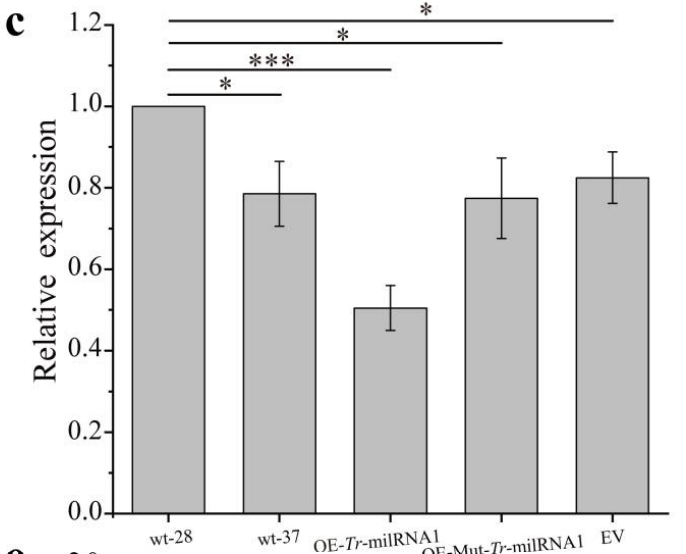

e

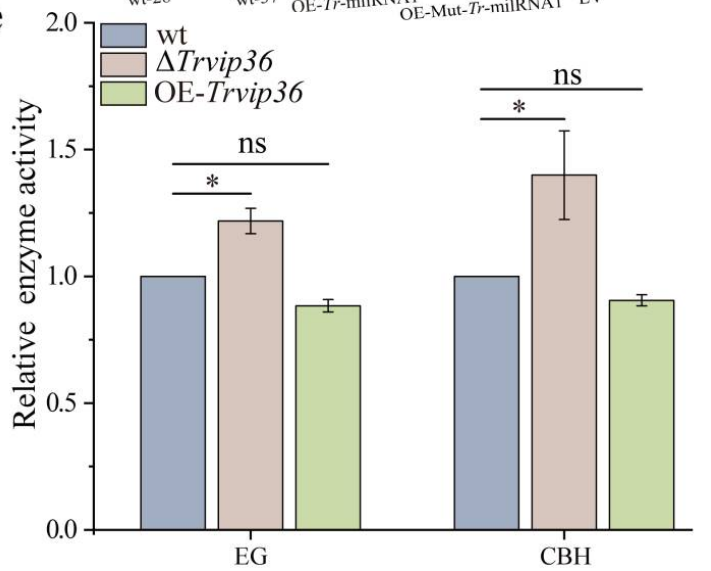

Figure 3. The function of Tr-milRNA1 in regulating the target gene Trvip36. (a) The precursor of Tr-milRNA1 could form a hairpin structure. The red line indicates the sequence of mature Tr-milRNA1; (b) alignment of Tr-milRNA1 and mutated Tr-milRNA1 (OE-Mut-Tr-milRNA1) with target gene (Trvip36) at the predicted binding sites; (c) relative expression of Trvip36 in wt at $28^{\circ} \mathrm{C}$, and in wt, OE-Tr-milRNA1, OE-Mut-Tr-milRNA1 and EV at $37^{\circ} \mathrm{C}$. Relative expression of Trvip36 was normalized to the Tef gene and calibrated to the levels of wt at $28^{\circ} \mathrm{C}$ (set as 1.0). Data were calculated from three biological replicates, and error bars represent \pm SDs. ${ }^{*} p<0.05,{ }^{* * *} p<0.001$. A $p$ value $<0.05$ was regarded as statistically significant and ns refers to no significance; (d) growth of wt and different mutants on PDA at $28^{\circ} \mathrm{C}$ (top row) and rice straw medium at $37^{\circ} \mathrm{C}$ (bottom row), respectively; (e) relative activities of EG and CBH between wt, $\triangle$ Trvip36 and OE-Trvip36 by using rice straw as the sole carbon source under heat stress. 


\subsection{Deletion of Trvip36 Improved the Secretion of Lignocellulases of NJAU 4742 under Heat Stress}

Deletion $(\Delta$ Trvip36) and overexpression (OE-Trvip36) strains of Trvip36 were generated to determine the function of lignocellulose utilization under heat stress. Compared to $\mathrm{wt}$, both mutants showed no difference in vegetative growth (Figure 3d). Nevertheless, $\Delta$ Trvip36 and OE-Trvip36 exhibited opposite growth trends, and $\Delta$ Trvip36 showed better growth rate relative to wt by using rice straw as the sole carbon source under heat stress (Figure 3d). Previous results showed that the EG and CBH activities increased significantly in OE-Tr-milRNA1. Thus, these two enzyme activities were also measured in $\Delta \operatorname{Tr} v i p 36$ and OE-Trvip36 to determine whether these changes were associated with the expression level of Trvip36. In correlation with Trvip36, EG and CBH activities increased significantly in $\Delta$ Trvip36 and slightly decreased in OE-Trvip36 (Figure 3e). These findings suggested that activation of Trvip36 might suppress lignocellulase secretion, and we concluded that impaired expression of Trvip36 regulated by Tr-milRNA1 contributes to the utilization of lignocellulose under heat stress.

\subsection{Deletion of Trvip36 Reduces the Retention of Lignocellulases in the ER of NJAU 4742 under Heat Stress}

In mammalian cells, VIP36 dynamically localized to the cis-Golgi and ER-Golgi intermediate compartment, and modification of its N-linked carbohydrate together with other evidence suggested that VIP36 possessed a role in trafficking through later Golgi compartments [32,33]. VIPL, a VIP36-like protein, is widely expressed in animal cells and mainly localizes to the ER [34]. In filamentous fungi, VIP36 was also found to localize in the ER and Golgi compartments and a higher fraction of VIP36 co-localized with the Golgi marker (Golgi protein AoGrh1 fused to EGFP) than with the ER marker (the ER membrane protein AoClxA fused to EGFP) [19]. We inferred that they might interfere with the normal intracellular transport of cellulases. Thus, strains expressing egl-GFP or $c b h$-GFP fusion proteins were generated in wt and $\Delta$ Trvip36 genetic backgrounds to track their intracellular localization. Cellulases tagged with eGFP were successfully expressed in hyphae of different mutants. To elucidate the aggregation level of cellulases in the ER and Golgi apparatus, the fluorescence intensities of EGL-GFP and CBH-GFP were detected in the ER and Golgi apparatus after staining with ER-Tracker ${ }^{\mathrm{TM}}$ Red and BODIPYTM TR Ceramide, respectively (Figure 4a,b,e,f). EGL-GFP and CBH-GFP intensities were analyzed in each pixel by ImageJ software. The average intensity of GFP fluorescence was calculated to measure the expression levels of EGL-GFP and CBH-GFP in the ER and Golgi apparatus. In $w t$, the average intensity of EGL-GFP and CBH-GFP was $20.13 \pm 1.70$ and $31.65 \pm 1.32$ in the ER, $50.29 \pm 1.60$ and $21.71 \pm 2.03$ in the Golgi apparatus, respectively. Compared to $\mathrm{wt}, \triangle$ Trvip36 exhibited lower average intensities of EGL-GFP $(15.03 \pm 0.99)$ and CBH-GFP (17.21 \pm 0.94$)$ fluorescence in the ER $(p<0.01)$. In contrast, higher average fluorescence intensities levels of EGL-GFP $(86.85 \pm 4.01)$ and CBH-GFP $(69.09 \pm 3.18)$ were distributed in the Golgi apparatus of $\Delta \operatorname{Trvip} 36(p<0.001)$ (Figure $4 \mathrm{c}, \mathrm{d}, \mathrm{g}, \mathrm{h}$ and Figure S4). These results suggest that VIP36 could interfere with the secretion of EGL and CBH by increasing the ER retention rate, which is consistent with the increased secretion of EGL and CBH in $\Delta$ Trvip36. 


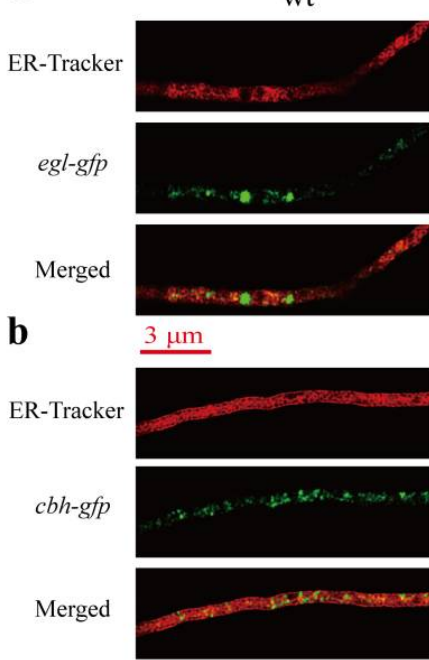

e

Golgi-Tracker

$e g l-g f p$
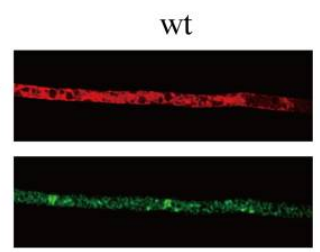

Merged
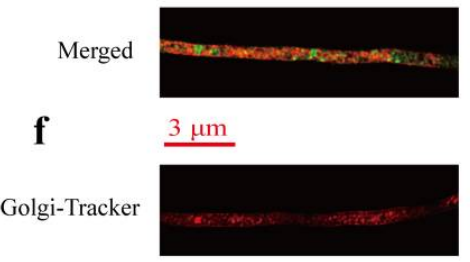

cbh-gfp

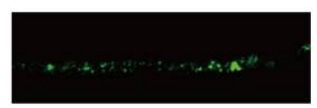

Merged

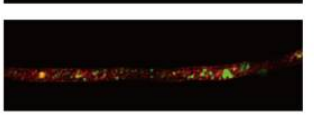

$\Delta \operatorname{Trvip} 36$
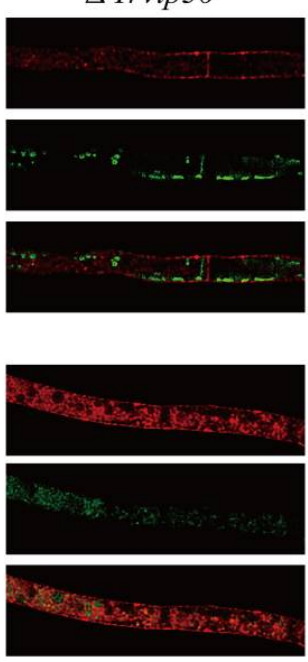

$\Delta$ Trvip36
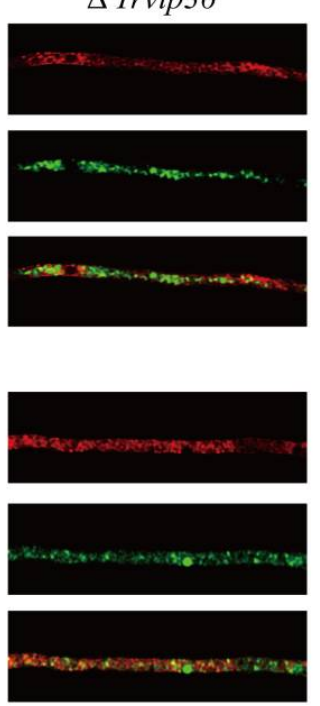

c
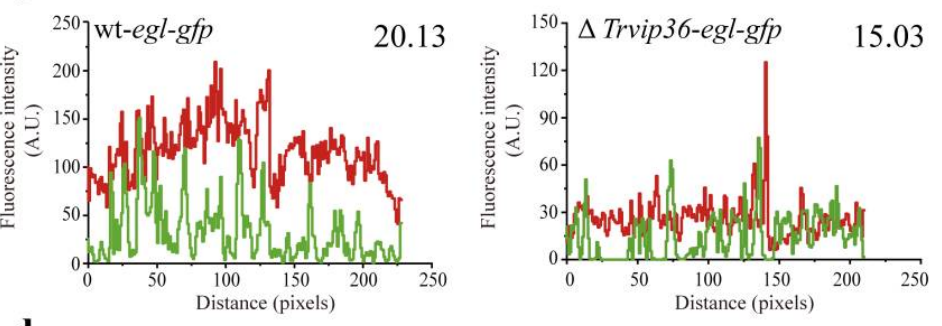

d
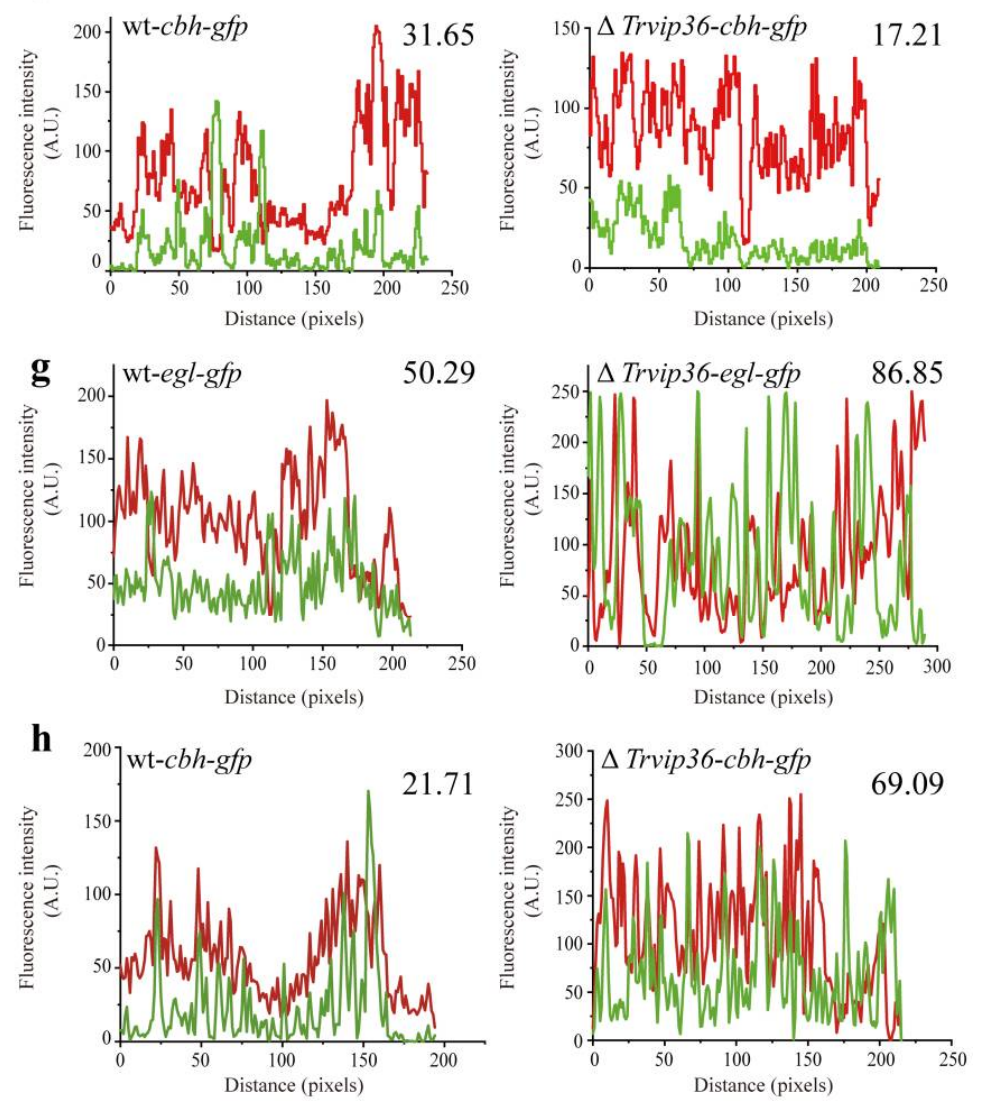

Figure 4. Localization analysis of EGL-GFP and CBH-GFP in wt and $\triangle$ Trvip36 genetic backgrounds after staining hyphae with ER-Tracker ${ }^{\mathrm{TM}}$ and BODIPY ${ }^{\mathrm{TM}}$ TR Ceramide, as markers of ER and Golgi compartments. (a,b) Confocal images of hyphae from egl-GFP and $c b h$-GFP fusion strains after staining with ER-Tracker ${ }^{\mathrm{TM}}$ Red to track endoglucanase and cellobiohydrolase localization in the ER between wt and $\Delta \operatorname{Trvip} 36$. Bar $=3 \mu \mathrm{m} ;(\mathbf{c}, \mathbf{d})$ the fluorescence intensity of the two channels (ER-Tracker (red) and GFP (green)) detected in wt and $\Delta$ Trvip36; (e,f) confocal images of hyphae from the egl-GFP and $c b h$-GFP fusion strains after staining with BODIPY ${ }^{\mathrm{TM}}$ TR ceramide to track endoglucanase and cellobiohydrolase localization in the Golgi apparatus between wt and $\Delta \operatorname{Trvip} 36 . \mathrm{Bar}=3 \mu \mathrm{m} ;(\mathrm{g}, \mathbf{h})$ the fluorescence intensity of the two channels (Golgi-Tracker (red) and GFP (green)) detected in wt and $\Delta$ Trvip36.

\section{Discussion}

Normally, most soil fungi experience a constantly fluctuating environment. Throughout the evolution process, fungi have developed different modes of reproduction and the ability to adapt to complex environments [35]. Generally, fungi operate in narrower temperature ranges, and a decrease in temperature may cause fungal dormancy, while an increase in temperature would lead to the apoptosis of fungi [36]. In fact, a substantial increase in temperature causes attenuation and eventually leads to the death of many organism [37]. The changes associated with heat stress responded to a set of proteins that promoted the survival of the organism. A major portion of these proteins were termed heat-shock proteins (Hsps). Hsps play a major role in cell cycle progression, replication, transcription and 
post-translational processes, including protein folding, stability, transportation and degradation, and they were also reported to activate many key signal transducers in fungi [38,39]. Therefore, filamentous fungi could precisely regulate the expression of specific genes to maintain essential biological functions under high temperature environment.

In our previous study, we found that cellulase secretion in strain NJAU 4742 was largely limited under heat stress during the SSF process, which seriously inhibited its ability to obtain carbon source. In addition, through proteomic analysis, it was found that some differentially expressed genes responded to temperature changes to regulate cellulase secretion (unpublished data). In this study, we first reported that Tr-milRNA was involved in the regulation of cellulase secretion under heat stress. It is generally accepted that miRNAs from plants are integral components of plant responses to disadvantageous environmental conditions [40,41]. For example, in Arabidopsis, miR398 was shown to be quickly induced under heat stress, accompanied by the downregulation of its target genes in response to heat stress [42]. Similarly, miR156 could accelerate the sustained expression of heat stress responsive genes through SPL genes [43]. Here, Tr-milRNA1, from filamentous fungi NJAU 4742, was revealed to be related to cellulase secretion under heat stress during SSF by regulating its target gene Trvip36.

Although numerous milRNAs have been identified in fungi [44,45], no homologous miRNAs were found in NJAU 4742 by mapping the clean reads of Tr-milRNA1 to miRBase 22.0. A similar result was discovered in T. reesei and F. oxysporum, which indicated a large degree of species specificity of fungal milRNAs [46]. More interestingly, there have been few relevant studies on the regulation of cellulase secretion by milRNAs, especially under heat stress. Previously, differential expression of milRNAs in T. reesei was discovered when T. reesei was cultivated in Avicel medium or glucose medium, which implied that milRNA might be involved in fungal growth and cellulase secretion [14]. In Ganoderma lucidum, several milRNA target genes were also identified by high-throughput sequencing and degradome analysis, and were considered glycoside hydrolases involved in the biosynthesis of polysaccharides [47]. Thus, it seemed that milRNAs were related to the regulation of cellulose degradation but there was a lack of in-depth evidence. In plants and animals, miRNAs could play vital regulatory roles by targeting mRNAs for cleavage or translational repression [10], while the regulatory mechanism of milRNAs in fungi is largely unknown. In this study, $T r$-milRNA1 negatively regulated the gene expression of lectin-type cargo receptor by suppressing the expression of Trvip36. There was evidence that Tr-milRNA1 regulated target gene expression in a sequence specific mode. During SSF, upregulation of Tr-milRNA1 increased the repression effect of Trvip36, which promoted cellulase secretion and exhibited better growth rate under heat stress. Deletion mutant of Trvip36 also showed a significant increase in cellulosic enzyme activities, which might be due to the reduction in cellulase retention in the ER compared to wt. Consistent with this speculation, in Aspergillus oryzae, AoVip36 was shown to have a protein retention function in the ER and the deletion of AoVip36 could increase the secretion of heterologous proteins [19]. In mammals, glycoprotein $\alpha 1$-antitrypsin was determined to be the retention target of VIP36, and it exhibited a significant promotion of intracellular transport upon silencing VIP36 [18]. VIP36 could also form a complex with $\mathrm{BiP}$ independently to increase its $\mathrm{N}$-glycan binding activity, and overproduction of a lectin-deficient form of VIP36 could decrease the secretion of clusterin [17]. These results suggested that VIP36 could also negatively interfere with protein transport in an N-glycan-independent manner [48]. In addition, it was reported that VIP36 could be recycled between the Golgi and the ER and carry out the retrograde transport of glycoproteins [49]. Moreover, it is worth noting that heat and nutrient deprivation stress could lead to the generation of protein aggregates in the ER [50]. Avoiding the excessive aggregation of proteins in the ER could alleviate ER stress, which would be conducive to protein synthesis and secretion [51]. Thus, we speculated that deletion of Trvip36 might also contribute to reducing the retrograde transport of lignocellulases and preventing the aggregation of cellulases in the ER to relieve ER stress thereby increasing the secretion of cellulases by using rice straw as the sole carbon source under heat stress. In addition, it 
was reported that deletion of VIP36 would impair the secretion of $\alpha$-amylase [19,52], which might suggest that some unnecessary pathways would be decreased to avoid excessive energy consumption of filamentous fungi under stress (Figure 5). In fact, the main component of rice straw is lignocellulose [53], and producing more lignocellulases is the most important way to obtain energy and maintain vegetative growth, especially under heat stress. However, its internal regulatory mechanism between Trvip36 and the secretion of $\alpha$-amylase in NJAU 4742 still needs in-depth and detailed study in the future.

Overexpression of T37 up-regulated $\operatorname{Tr}$-milRNAs

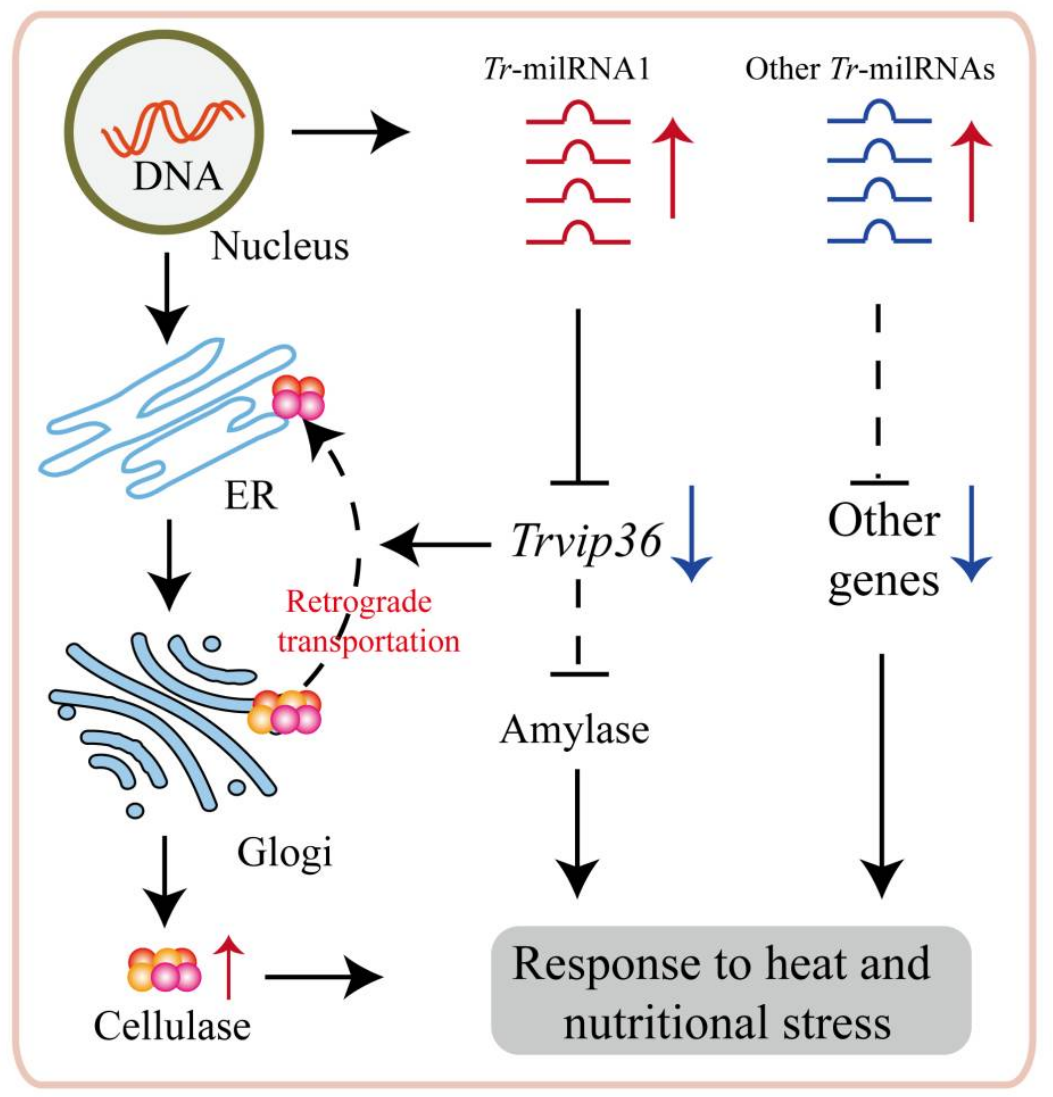

Figure 5. Proposed model for the regulation of NJAU 4742 by Tr-milRNAs during the lignocellulose utilization process under heat stress. Tr-milRNA1 is involved in regulating cellulase secretion by revoking the expression of Trvip36 to decrease the cellulase retrograde transportation.

\section{Conclusions}

Overall, this study demonstrated that Tr-milRNA1 from NJAU 4742 played a critical role in cellulase secretion by regulating the endogenous target gene Trvip36. These results provide important evidence to determine the roles of milRNA and their corresponding target gene during the utilization of lignocellulose in filamentous fungi under heat stress.

Supplementary Materials: The following are available online at https:/ / www.mdpi.com/article/10 .3390/jof7120997/s1, Figure S1: Knockout or over-expression of Tr-milRNA or gene in NJAU 4742. (a) Schematic diagram for Tr-milRNA or gene disruption by double crossover recombination; (b) schematic diagram for Tr-milRNA over-expression; (c) schematic diagram for gene over-expression; (d) analysis of expression level of $T r$-milRNA relative to $18 \mathrm{~S}$ in wt, OE-Tr-milRNA1, OE-Mut- $T r$ milRNA1, and empty vector transformant (EV) strains determined by qPCR. (e) analysis of expression level of Trvip36 gene relative to Tef in wt and OE-Trvip36 strain determined by qPCR. Data were calculated from three biological replicates. Error bars represent \pm SDs. ${ }^{*} p<0.05,{ }^{* *} p<0.01$, *** $p<0.001$. A $P$-value $<0.05$ was regarded as statistically significant. The expression values are normalized to wt; (f,g) verification of $\Delta T r$-milRNA1 (f), $\Delta$ Trvip36 (g), wt-egl-GFP, $\Delta$ Trvip36-egl-GFP, wtcbh-GFP, and $\Delta$ Trvip36-cbh-GFP (h) mutants by PCR to verify homologous recombination. Figure S2: 
Diagram of construction principle for prepaing the mutants of lignocellulases-eGFP fusion strains. Arm 1 and Arm 2 were used as two arms of homologous recombination and Hygb gene was used as the biomaker for screening. Figure S3: GO (a) and KEGG (b) enrichment of candidate Tr-milRNAs target genes. Figure S4: The average intensity of EGL-GFP and CBH-GFP fluorescence in the ER and Golgi apparatus of wt and $\Delta$ Trvip36 after staining hyphae with ER-Tracker ${ }^{\mathrm{TM}}$ and BODIPY ${ }^{\mathrm{TM}} \mathrm{TR}$ Ceramide, as markers of ER and Golgi compartments. Data were calculated from all valid pixels in the ER and Golgi apparatus, and error bars represent \pm SEs. ${ }^{*} p<0.05,{ }^{* *} p<0.01,{ }^{* * *} p<0.001$. A $p$ value $<0.05$ was regarded as statistically significant and ns refers to no significance. Table S1: All PCR primers used in this study.

Author Contributions: T.L. (Tuo Li) performed the majority of the experiments and wrote the manuscript; J.L. analyzed the sequencing data of Tr-milRNAs; Q.W. determined the lignocellulases activities; Y.L. constructed the mutant OE-Tr-milRNA1; T.L. (Ting Li) constructed the mutant $\Delta$ Trvip36; D.L. and Q.S. assisted in the drafting and revision of the manuscript. D.L. was the corresponding author. All authors have read and agreed to the published version of the manuscript.

Funding: This research was financially supported by National Natural Science Foundation of China (31972513), the National Key R \& D Program of China (2018YFD0500201), and the Fundamental Research Funds for the Central Universities (KYZ201716).

Institutional Review Board Statement: Not applicable.

Informed Consent Statement: Not applicable.

Data Availability Statement: The data that support the findings of this study areavailable on request from the corresponding author.

Acknowledgments: We gratefully acknowledge all fundings for supporting this research work.

Conflicts of Interest: The authors declare no conflict of interest. The funders had no role in the design of the study; in the collection, analyses or interpretation of data; in the writing of the manuscript or in the decision to publish the results.

\section{References}

1. Chang, S.S.; Zhang, Z.; Liu, Y. RNA Interference Pathways in Fungi: Mechanisms and Functions. Annu. Rev. Microbiol. 2012, 66, 305-323. [CrossRef]

2. Castel, S.E.; Martienssen, R.A. RNA interference in the nucleus: Roles for small RNAs in transcription, epigenetics and beyond. Nat. Rev. Genet. 2013, 14, 100-112. [CrossRef] [PubMed]

3. Holoch, D.; Moazed, D. RNA-mediated epigenetic regulation of gene expression. Nat. Rev. Genet. 2015, 16, 71-84. [CrossRef]

4. Zhang, T.; Zhao, Y.L.; Zhao, J.H.; Wang, S.; Jin, Y.; Chen, Z.Q.; Fang, Y.Y.; Hua, C.L.; Ding, S.W.; Guo, H.S. Cotton plants export microRNAs to inhibit virulence gene expression in a fungal pathogen. Nat. Plants 2016, 2, 16153. [CrossRef] [PubMed]

5. Li, L.; Chang, S.S.; Liu, Y. RNA interference Pathways in Filamentous Fungi. Cell. Mol. Life Sci. 2010, 67, 3849-3863. [CrossRef]

6. Romano, N.; Macino, G. Quelling: Transient inactivation of gene expression in Neurospora crassa by transformation with homologous sequences. Mol. Microbiol. 1992, 6, 3343-3353. [CrossRef]

7. Verdel, A.; Moazed, D. RNAi-directed assembly of heterochromatin in Fission yeast. FEBS Lett. 2005, 579, 5872-5878. [CrossRef]

8. Carthew, R.W.; Sontheimer, E.J. Origins and mechanisms of miRNAs and siRNAs. Cell 2009, 136, 642-655. [CrossRef]

9. Mallory, A.C.; Vaucheret, H. Functions of microRNAs and related small RNAs in plants. Nat. Genet. 2006, 38, S31-S36. [CrossRef] [PubMed]

10. Bartel, D.P. MicroRNAs: Genomics, Biogenesis, Mechanism, and Function. Cell 2004, 116, 281-297. [CrossRef]

11. Lee, H.C.; Li, L.; Gu, W.; Xue, Z.; Yi, L. Diverse Pathways Generate MicroRNA-like RNAs and Dicer-Independent Small Interfering RNAs in Fungi. Mol. Cell 2010, 38, 803-814. [CrossRef]

12. Zhou, Q.; Wang, Z.; Zhang, J.; Meng, H.; Huang, B. Genome-wide identification and profiling of microRNA-like RNAs from Metarhizium anisopliae during development. Fungal Biol. 2012, 116, 1156-1162. [CrossRef]

13. Weiberg, A.; Wang, M.; Lin, F.M.; Zhao, H.; Zhang, Z.; Kaloshian, I.; Huang, H.D.; Jin, H. Fungal Small RNAs Suppress Plant Immunity by Hijacking Host RNA Interference Pathways. Science 2013, 342, 118-123. [CrossRef]

14. Kang, K.; Zhong, J.; Jiang, L.; Liu, G.; Gou, C.Y.; Wu, Q.; Wang, Y.; Luo, J.; Gou, D. Identification of microRNA-Like RNAs in the filamentous fungus Trichoderma reesei by solexa sequencing. PLoS ONE 2013, 8, e76288. [CrossRef]

15. Jin, Y.; Zhao, J.H.; Zhao, P.; Zhang, T.; Wang, S.; Guo, H.S. A fungal milRNA mediates epigenetic repression of a virulence gene in Verticillium dahliae. Philos. Trans. R. Soc. Lond. Ser. B Biol. Sci. 2019, 374, 20180309. [CrossRef]

16. Druzhinina, I.S.; Seidl-Seiboth, V.; Herrera-Estrella, A.; Horwitz, B.A.; Kenerley, C.M.; Monte, E.; Mukherjee, P.K.; Zeilinger, S.; Grigoriev, I.V.; Kubicek, C.P. Trichoderma: The genomics of opportunistic success. Nat. Rev. Microbiol. 2011, 9, 749-759. [CrossRef] [PubMed] 
17. Daisuke, N.; Osamu, S.; Norihito, K.; Naoki, M.; Kazuo, Y. Stable interaction of the cargo receptor VIP36 with molecular chaperone BiP. Glycobiology 2007, 17, 913-921.

18. Reiterer, V.; Nyfeler, B.; Hauri, H.P. Role of the lectin VIP36 in post-ER quality control of human alpha1-antitrypsin. Traffic 2010, 11, 1044-1055. [CrossRef]

19. Hoang, H.D.; Maruyama, J.I.; Kitamoto, K.; Brakhage, A. Modulating Endoplasmic Reticulum-Golgi Cargo Receptors for Improving Secretion of Carrier-Fused Heterologous Proteins in the Filamentous Fungus Aspergillus oryzae. Appl. Environ. Microbiol. 2015, 81, 533-543. [CrossRef]

20. Miao, J.; Wang, M.; Ma, L.; Li, T.; Huang, Q.; Liu, D.; Shen, Q. Effects of amino acids on the lignocellulose degradation by Aspergillus fumigatus Z5: Insights into performance, transcriptional, and proteomic profiles. Biotechnol. Biofuels 2019, 12, 1-19. [CrossRef] [PubMed]

21. Ana, K.; Sam, G.J. miRBase: Integrating microRNA annotation and deep-sequencing data. Nucleic Acids Res. 2011, 39, D152-D157.

22. Mackowiak, S.D. Identification of novel and known miRNAs in deep-sequencing data with miRDeep2. Curr. Protoc. Bioinform. 2011, 36, 12.10.1-12.10.15. [CrossRef]

23. Bo, X.; Wang, S. TargetFinder: A software for antisense oligonucleotide target site selection based on MAST and secondary structures of target mRNA. Bioinformatics 2005, 21, 1401-1402. [CrossRef]

24. Dai, X.; Zhao, P.X. psRNATarget: A plant small RNA target analysis server. Nucleic Acids Res. 2011, 39, W155-W159. [CrossRef]

25. Varkonyi-Gasic, E.; Wu, R.; Wood, M.; Walton, E.F.; Hellens, R.P. Protocol: A highly sensitive RT-PCR method for detection and quantification of microRNAs. Plant Methods 2007, 3, 12. [CrossRef]

26. Zhang, J.; Miao, Y.; Rahimi, M.J.; Zhu, H.; Steindorff, A.; Schiessler, S.; Cai, F.; Pang, G.; Chenthamara, K.; Xu, Y. Guttation capsules containing hydrogen peroxide: An evolutionarily conserved NADPH oxidase gains a role in wars between related fungi. Environ. Microbiol. 2019, 21, 2644-2658. [CrossRef]

27. Xue, D.; Lin, D.; Gong, C.; Peng, C.; Yao, S. Expression of a bifunctional cellulase with exoglucanase and endoglucanase activities to enhance the hydrolysis ability of cellulase from a marine Aspergillus niger. Process Biochem. 2017, 52, 115-122. [CrossRef]

28. Miao, Y.; Kong, Y.; Li, P.; Li, G.; Liu, D.; Shen, Q.; Zhang, R. Effect of CBM1 and linker region on enzymatic properties of a novel thermostable dimeric GH10 xylanase (Xyn10A) from filamentous fungus Aspergillus fumigatus Z5. AMB Express 2018, 8, 1-10. [CrossRef]

29. Liu, D.; Li, J.; Zhao, S.; Zhang, R.; Wang, M.; Miao, Y.; Shen, Y.; Shen, Q. Secretome diversity and quantitative analysis of cellulolytic Aspergillus fumigatus Z5 in the presence of different carbon sources. Biotechnol. Biofuels 2013, 6, 1-16. [CrossRef]

30. Zhao, X.; Yong, F.; Yang, Y.; Yu, Q.; Ding, M. Elaiophylin, a novel autophagy inhibitor, exerts antitumor activity as a single agent in ovarian cancer cells. Autophagy 2015, 11, 1849-1863. [CrossRef] [PubMed]

31. Wi, S.G.; Cho, E.J.; Lee, D.S.; Lee, S.J.; Lee, Y.J.; Bae, H.J. Lignocellulose conversion for biofuel: A new pretreatment greatly improves downstream biocatalytic hydrolysis of various lignocellulosic materials. Biotechnol. Biofuels 2015, 8, 228. [CrossRef] [PubMed]

32. Hara-Kuge, S. Involvement of VIP36 in Intracellular Transport and Secretion of Glycoproteins in Polarized Madin-Darby Canine Kidney (MDCK) Cells. J. Biol. Chem. 2002, 277, 16332. [CrossRef] [PubMed]

33. Shimada, O.; Hara-Kuge, S.; Yamashita, K.; Tosaka-Shimada, H.; Yanchao, L.; Einan, L.; Atsumi, S.; Ishikawa, H. Localization of VIP36 in the post-Golgi secretory pathway also of rat parotid acinar cells. J. Histochem. Cytochem. Off. J. Histochem. Soc. 2003, 51, 1057-1063. [CrossRef] [PubMed]

34. Neve, E.; Svensson, K.; Fuxe, J.; Pettersson, R.F. VIPL, a VIP36-like membrane protein with a putative function in the export of glycoproteins from the endoplasmic reticulum. Exp. Cell Res. 2003, 288, 70-83. [CrossRef]

35. Lamoth, F.; Juvvadi, P.R.; Steinbach, W.J. Heat shock protein 90 (Hsp90) in fungal growth and pathogenesis. Curr. Fungal Infect. Rep. 2014, 8, 296-301. [CrossRef]

36. Lamoth, F.; Juvvadi, P.R.; Fortwendel, J.R.; Steinbach, W.J. Heat shock protein 90 is required for conidiation and cell wall integrity in Aspergillus fumigatus. Eukaryot. Cell 2012, 11, 1324-1332. [CrossRef]

37. Bhabhra, R.; Askew, D. Thermotolerance and virulence of Aspergillus fumigatus: Role of the fungal nucleolus. Med. Mycol. 2005, 43, S87-S93. [CrossRef]

38. Kregel, K.C. Invited Review: Heat shock proteins: Modifying factors in physiological stress responses and acquired thermotolerance. J. Appl. Physiol. 2002, 192, 2177-2186. [CrossRef]

39. Verghese, J.; Abrams, J.; Wang, Y.; Morano, K.A. Biology of the Heat Shock Response and Protein Chaperones: Budding Yeast (Saccharomyces cerevisiae) as a Model System. Microbiol. Mol. Biol. Rev. 2012, 76, 115-158. [CrossRef]

40. Katiyar-Agarwal, S.; Jin, H. Role of Small RNAs in Host-Microbe Interactions. Annu. Rev. Phytopathol. 2010, 48, 225. [CrossRef]

41. Li, S.; Castillo-Gonzalez, C.; Yu, B.; Zhang, X. The functions of plant small RNAs in development and in stress responses. Plant J. 2017, 90, 654. [CrossRef]

42. Guan, Q.; Lu, X.; Zeng, H.; Zhang, Y.; Zhu, J. Heat stress induction of miR398 triggers a regulatory loop that is critical for thermotolerance in Arabidopsis. Plant J. 2013, 74, 840-851. [CrossRef] [PubMed]

43. Cui, L.G.; Shan, J.X.; Shi, M.; Gao, J.P.; Lin, H.X. The miR156-SPL 9-DFR pathway coordinates the relationship between development and abiotic stress tolerance in plants. Plant J. 2014, 80, 1108-1117. [CrossRef] [PubMed]

44. Xu, M.; Guo, Y.; Tian, R.; Gao, C.; Guo, F.; Voegele, R.T.; Bao, J.; Li, C.; Jia, C.; Feng, H. Adaptive regulation of virulence genes by microRNA-like RNAs in Valsa mali. New Phytol. 2020, 227, 899-913. [CrossRef] 
45. Feng, H.; Xu, M.; Gao, Y.; Liang, J.; Guo, F.; Guo, Y.; Huang, L. Vm-milR37 contributes to pathogenicity by regulating glutathione peroxidase gene VmGP in Valsa mali. Mol. Plant Pathol. 2021, 22, 243-254. [CrossRef]

46. Chen, R.; Jiang, N.; Jiang, Q.; Sun, X.; Wang, Y.; Zhang, H.; Hu, Z. Exploring microRNA-like small RNAs in the filamentous fungus Fusarium oxysporum. PLoS ONE 2014, 9, e104956.

47. Js, A.; Lw, A.; Yang, L.B.; Qq, B.; Bw, B.; Sl, A.; Chang, L.A. Identification of milRNAs and their target genes in Ganoderma lucidum by high-throughput sequencing and degradome analysis. Fungal Genet. Biol. 2020, 136, 103313.

48. O'Keefe, S. Small Molecule Inhibitors Targeting Protein Biogenesis at the Endoplasmic Reticulum; The University of Manchester: Manchester, UK, 2019.

49. Kamiya, Y.; Kato, K. Sugar Recognition by Intracellular Lectins That Determine the Fates of Glycoproteins. Trends Glycosci. Glycotechnol. 2006, 18, 231-244. [CrossRef]

50. Tyedmers, J.; Mogk, A.; Bukau, B. Cellular strategies for controlling protein aggregation. Nat. Rev. Mol. Cell Biol. 2010, 11, 777-788. [CrossRef] [PubMed]

51. Buchberger, A.; Bukau, B.; Sommer, T. Protein quality control in the cytosol and the endoplasmic reticulum: Brothers in arms. Mol. Cell 2010, 40, 238-252. [CrossRef]

52. Hara-Kuge, S.; Seko, A.; Shimada, O.; Tosaka-Shimada, H.; Yamashita, K. The binding of VIP36 and $\alpha$-amylase in the secretory vesicles via high-mannose type glycans. Glycobiology 2004, 14, 739-744. [CrossRef] [PubMed]

53. Gu, Y.; Chen, X.; Liu, Z.; Zhou, X.; Zhang, Y. Effect of inoculum sources on the anaerobic digestion of rice straw. Bioresour. Technol. 2014, 158, 149-155. [CrossRef] [PubMed] 